\title{
Análisis Químico de Mezclas Biodiesel de Aceite de Cocina Usado y Diesel por Espectroscopia Infrarroja
}

\author{
Jennifer J. Lafont ${ }^{\star}$, Manuel S. Páez y Yudi C. Torres \\ Universidad de Córdoba, Facultad de Ciencias Básicas e Ingenierías, Departamento de Química, \\ Carrera 6 No 76-103, Montería, Córdoba-Colombia (e-mail: jenniferlafontm@yahoo.es) \\ * autor a quien debe ser dirigida la correspondencia
}

Recibido Ago. 24, 2010; Aceptado Nov. 04, 2010; Versión Final recibida Ene. 03, 2011

\section{Resumen}

Se propone un método eficiente para determinar los porcentajes óptimos de mezclas biodiesel de aceite usado y diesel, para uso automotor. El procedimiento consiste en recolección del aceite usado, limpieza de residuos, y obtención del biodiesel mediante una reacción de transesterificación, con diferentes proporciones molares metanol/aceite. Las muestras fueron analizadas por espectroscopia infrarroja. Se encontró que una proporción metanol/aceite de 1:8 presentó mayor rendimiento en la obtención de biodiesel. Los espectros de infrarrojo mostraron el incremento del máximo de carbonilo de los esteres metílicos al aumentar las concentraciones de biodiesel en las mezcla biodiesel+diesel. Se concluye que la técnica usada cumple con la ley de Beer-Lambert y es adecuada para cuantificar este tipo de mezclas.

Palabras claves: mezclas biodiesel/diesel, aceite de cocina usado, espectroscopia infrarrojo, propiedades fisicoquímicas.

\section{Chemical Analysis of Mixtures of Biodiesel from Used Cooking Oil and Diesel by Infrared Spectroscopy}

\begin{abstract}
An efficient method to determine the optimum percentages of blends biodiesel from used oil and diesel for automotive use, is proposed. The procedure consists of recollection of used frying oil, residue cleaning and synthesis of biodiesel by a transesterification reaction with different molar proportions of methanol/oil. The samples were analyzed by infrared spectroscopy. It was found that a ratio methanol/oil of 1:8 showed higher performance in the production of biodiesel. The infrared spectra showed an increase in the carbonyl peak of the methyl esters with the increase of the concentration of biodiesel in the mixtures biodiesel+diesel. It is concluded that proposed technique complies with the Beer-Lambert law and it is a good method to quantify these types of mixtures.
\end{abstract}

Keywords: blends biodiesel/diesel, Infrared spectroscopy, frying oil, physicochemical properties 


\section{INTRODUCCIÓN}

La disminución de las reservas de petróleo y los diferentes problemas ambientales generados por los combustibles fósiles, entre otras causas, ha generado la búsqueda de nuevas fuentes de energía; destacandose el biodiesel, que es una mezcla de ésteres alquilicos, producido a través de una reacción de transesterificación, entre un aceite vegetal o grasa animal y un alcohol de cadena corta para producir los esteres respectivos y el subproducto glicerol (Helwani et al., 2009; Leuny, 2010). Actualmente se está produciendo el biodiesel a partir de diversas materias primas (Marticorena et al., 2010) como soya, girasol, palma y jatropha, entre otras, las cuales son también utilizadas industrialmente para la extracción y comercialización de aceites comestibles, representando un gran limitante para la producción de este combustible (Kulkarn y Dalai., 2006) en razón a lo anterior, las investigaciones están siendo dirigidas hacia la búsqueda de materias primas alternativas, que produzcan un combustible eficiente, sin afectar la alimentación y que sea económicamente viable. Una alternativa la constituye los residuos de aceite de cocina usado en frituras, por cuanto ellos han demostrado ser una fuente económica para la producción de biodiesel, en virtud a su alta disponibilidad y bajo costo, además su reciclaje y reutilización como forma de energía renovable, disminuye el poder contaminante de estos residuos al ser vertidos en los fregaderos. (Wang et al., 2007; Demirbas, 2009).

El biodiesel es considerado como el mejor sustituto del diesel y puede ser utilizado puro o en mezclas biodiesel /diesel, presentando ventajas ambientales, economicas, sociales y técnicas (Guarieiro et al., 2008) Entre las normas mas utilizadas que rigen la calidad del biodiesel se encuentran la ASTM D 975 (American Society of Testing and Materials) y EN 590 (Norma Europea), donde establecen valores límite permitidos para cada parámetro fisicoquímico, que predicen la eficiencia del combustible en el motor (EN-590:2004; ASTM D 975: 2006). Además de los procedimientos experimentales; tambien se pueden determinar simultáneamente algunos parámetros de calidad de mezclas biodiesel/diesel a través de la técnica del Infrarrojo aplicando regresiones de mínimos cuadrados parciales, como gravedad específica, punto de inflamación y contenido de sulfuro (Flores et al., 2011).

Dado el incremento en el uso de biodiesel en el mundo, muchos estudios se han enfocado en desarrollar métodologías para cuantificar los porcentajes de mezclas biodiesel/diesel suministrados en estaciones de distribución del combustible; empleándose técnicas como la cromatografía de gases acoplada a masas (Dardot et al., 2010), cromatografía líquida de alta eficiencia (HPLC) (Monteiro et al., 2008; Enweremadu, 2009), resonancia nuclear protónica (RMN${ }^{1} \mathrm{H}$ ) (Monteiro et al., 2009) las cuales resultan costosas, en comparación con otras técnicas de más fácil acceso, como la espectroscopia infrarroja con transformada de fourier, la cual toma como patrón la diferencia en los espectros del diesel y biodiesel observandose en el último el incremento en la absorción del pico intenso que representa al grupo carbonilo entre (1600 y $1700) \mathrm{cm}^{-1}$ a medida que aumenta la concentración del biodiesel en las mezclas respectivas (Aliske et al. 2007; Aryee, 2009).

Nuestro interés en este trabajo consiste en elaborar biodiesel a partir de aceite de cocina usado y con él, realizar un patrón de concentraciones de mezclas biodiesel/diesel en el rango de (0-100)\% que puedan ser fácilmente analizadas por la técnica de espectroscopia infrarroja, permitiendo la cuantificación de los porcentajes de mezclas suministrados en las estaciones de distribución del combustible; esta metodología ha sido ensayada en mezclas de diesel con biodiesel de soya, girasol, palma entre otros aceites menos comunes (Oliveira et al., 2006); sin embargo para el biodiesel de aceite de cocina usado hay muy pocos ensayos reportados (Aliske et al., 2007).

\section{METODOLOGÍA}

Se recolectaron muestras de aceite reutilizado en las cafeterías de la universidad de Córdoba y se sometieron a un tratamiento previo de limpieza. El procedimiento consistió en filtrar usando una malla de $5 \mu \mathrm{m}$ de poro y centrifugar a 5000rpm durante cinco minutos, con el fin de separar los residuos de alimentos presentes. El aceite libre de partículas se lavó con agua destilada a $70^{\circ} \mathrm{C}$, para eliminar las impurezas químicas (gomas); luego el agua fue separada por decantación; la 
humedad del aceite se eliminó con un rotavaporador a $90^{\circ} \mathrm{C}$ y presión reducida durante dos horas. Posteriormente se procedió a sintetizar el biodiesel mediante una reacción de transesterificación, con proporciones molares metanol/aceite de $(1: 3,1: 6,1: 8,1: 10)$, en presencia de hidróxido de potasio al $1 \% \mathrm{p} / \mathrm{p}$ como catalizador, a $65^{\circ} \mathrm{C}$ y $270 \mathrm{rpm}$ durante 1.5 horas a presión atmosférica, en un sistema de reflujo (Umer y Farooq., 2008). Este procedimiento, se realizó por triplicado. Completado el periodo de reacción se procedió a recuperar el metanol en exceso a presión reducida con la ayuda de una bomba de vacío a una temperatura de $50^{\circ} \mathrm{C}$ y $60 \mathrm{rpm}$ durante un periodo de tiempo de una hora; luego se dejó reposar durante 24horas para separar completamente las fases biodiesel/glicerol; la fase inferior correspondía al glicerol con un color café oscuro y la fase superior al biodiesel de color amarillo leve; la fase de glicerol se separó por decantación y se procedió a centrifugar el biodiesel a 5000rpm durante 5 minutos, retirando con esto las trazas de glicerol presente. El biodiesel se lavó con $10 \%$ de agua bidestilada a $50^{\circ} \mathrm{C}$ tres veces para cada proporción molar; luego se rotaevaporó al vacío a $90^{\circ} \mathrm{C}$ durante dos horas; finalmente sus productos fueron identificados por GC-MS. Una parte del biodiesel obtenido, se utilizó para realizar los análisis de las propiedades fisicoquímicas y la otra parte para preparar las mezclas biodiesel/ diesel.

La determinación de la viscosidad cinemática del biodiesel a $20^{\circ} \mathrm{C}$ se realizó usando un viscosímetro Ubbelohde. La calibración del viscosímetro se llevo a cabo a las temperaturas de 10 , $15,20,25,30$ y $35^{\circ} \mathrm{C}$ utilizando un control de temperatura marca LAUDA E-100 y un enfriador externo acoplado al termostato. Como liquido de calibración se usó agua bidestilada con una conductividad menor a $2 \mu \mathrm{S}$. Las variaciones de temperatura en el mismo se registraron con un termómetro Fluke Hart Scientific de alta precisión de $\pm 0.001^{\circ} \mathrm{C}$, el procedimiento se repitió 10 veces. Las medidas de densidad para el biodiesel a $15^{\circ} \mathrm{C}$ se realizaron utilizando un picnómetro de cuello capilar tipo Wood-Brusie con capacidad de $50 \mathrm{~mL}$; previa calibración del picnómetro con agua bidestilada a $10,15,20,25,30$ y $35^{\circ} \mathrm{C}$ (Giraldo et al., 2009). El número ácido (ASTM D 664) se determinó por medio de la titulación de la muestra con una solución estándar de hidróxido de potasio $0,01 \mathrm{M}$; la muestra fue preparada agregando $1 \mathrm{~g}$ de biodiesel en $10 \mathrm{~mL}$ de 2-metilpropanol; se utilizó fenolftaleína como indicador para determinar el punto final de la reacción. El punto de enfriamiento (ASTM D 2500) fue determinado por inspección óptica en un baño de agua a $-1^{\circ} \mathrm{C}$, para lo cual se introdujo la muestra de biodiesel en un tubo de ensayo y en el momento en que se observó la primera nube cristales, se determinó la temperatura que corresponde al punto de enfriamiento. Para la determinación del contenido de catalizador y jabón (ASTM D 2986) se disolvieron $10 \mathrm{~g}$ de la muestra en $100 \mathrm{~mL}$ de acetona con $2 \%$ de agua destilada, se añadió unas gotas de fenolftaleína y se procedió a titular la solución con ácido clorhídrico $0.01 \mathrm{M}$ hasta que se obtuvo el viraje de color; luego se añadió $1 \mathrm{~mL}$ de azul de bromofenol como indicador y se continuó con la valoración hasta que se observó el cambio de color de azul a amarillo.

Se prepararon $10 \mathrm{~g}$ de cada mezcla biodiesel/diesel, usando una balanza analítica OHAUS con una precisión de $0.0001 \mathrm{~g}$ a las concentraciones de: $(1,2,3,4,5,6,7,8,9,10,20,30,40,50,60$, $70,80,90$ y 100$) \% \mathrm{p} / \mathrm{p}$, denominadas $\mathrm{B} 1, \mathrm{~B} 2$, hasta B100 respectivamente, siendo este último el biodiesel sin mezcla. Para la corrosión de la tira de cobre (ASTM D 130) se tomaron las dieciocho mezclas de biodiesel/diesel y $\mathrm{B} 100$, se calentaron a $50^{\circ} \mathrm{C}$, se les adicionó una lámina de cobre pulida y se dejaron en calentamiento durante 3horas seguidas. Seguido se retiraron las láminas y se lavaron con etanol. Todos los procedimientos se realizaron por triplicado; con el propósito de evaluar el valor promedio y la desviación estándar $(\sigma)$ para cada análisis.

Los espectros infrarrojo se iniciaron con las muestras puras de biodiesel y diesel para establecer las diferencias entre ellos; luego se continuó con las dieciocho mezclas a las diferentes concentraciones; todos los espectros fueron realizados con un espectrofotómetro infrarrojo con transformada de Fourier, marca Perkin Elmer, modelo Spectrum BX, en el intervalo de longitudes de onda de $450 \mathrm{~cm}^{-1}$ a $4000 \mathrm{~cm}^{-1}$. Con la ayuda del software Spectrum $\vee 2.00$, se realizó la interpretación espectral y el análisis cuantitativo a traves de la regresion mínimos cuadrados clásicos (CLS), de mínimos cuadrados parciales (PLS) y análisis de los componentes principales (PCA); los algoritmos de procesos estandar suministran datos de absorbancias /transmitancia; áreas, alturas de pico, correccion de línea base, entre otros. 
Los valores así obtenidos de absorbancia y altura del pico carbonilo presente en los esteres metílicos, fueron relacionados con las concentraciones; mediante los gráficos de logaritmo de la altura de pico carbonilo (log $A_{p}$ )vs el logaritmo de la concentración (log C) y valores de absorbancia (A) vs concentración (C) de las mezclas de biodiesel de aceite de cocina usado/diesel. Los valores obtenidos fueron comparados con el método empleado por Aliske para biodiesel obtenidos de diferentes materias primas..

\section{RESULTADOS Y DISCUSIÓN}

De las proporciones molares estudiadas en la reacción de producción del biodiesel se encontró que la proporción 1:8 metanol/aceite fue la que presentó mayor rendimiento de reacción con un $92.046 \pm 0.641 \%$. A medida que aumenta el porcentaje de metanol, la producción de biodiesel se mejora significativamente. La proporción 1:10 es comparable con la 1:8, con un porcentaje de rendimiento del $91.310 \pm 0.712 \%$, que no justifica el mayor gasto de metanol, ya que se disminuye el rendimiento de la reacción, debido a que el exceso de metanol dificulta la separación del glicerol por decantación (Umer y Farooq et al., 2008). Las proporciones molares de 1:6 y 1:3 presentaron un rendimiento menor del $70 \%$, lo que se atribuye a que la reacción es incompleta. En otros estudios realizados con diversos tipos de aceites, se encontró la proporción 1:6 como la más adecuada para obtener altos rendimientos de producción, condición que no presentó el aceite de cocina usado que requirió un exceso de metanol para alcanzar altos rendimientos (véase tabla 1).

Tabla 1: Proporciones molares de la producción de biodiesel a partir de aceite de cocina usado.

\begin{tabular}{cc}
\hline Proporción molar (Metanol /aceite) & $\begin{array}{c}\text { Rendimiento Biodiesel (\%) } \\
\mathbf{x} \pm \sigma\end{array}$ \\
\hline $1: 3$ & $32.477 \pm 0.657$ \\
$1: 6$ & $67.915 \pm 1.110$ \\
$1: 8$ & $92.046 \pm 0.641$ \\
$1: 10$ & $91.310 \pm 0.712$ \\
\hline
\end{tabular}

El biodiesel obtenido fue analizado mediante la tecnica de GC-MS, cuyos esteres metilicos fueron identificados mediante la comparaciòn con el software del equipo National Institute of Standards and Technology (NIST). encontrandose los siguientes compuestos: Araquidato de metilo (C20:0), Linoleato de metilo (C18:2), Linolenato de metilo (C18:3), Oleato de metilo (C18:1), Palmitato de metilo (C16:0), Palmitoleato de Metilo (C16:1), Miristato de metilo (C14:0), Laureato de metilo (C12:0) Capriato de metilo (C10:0).

La tabla 2, muestra los resultados obtenidos de las propiedades fisicoquímicas para el biodiesel en términos de valor promedio y desviación estándar $x \pm \sigma$ en la última columna, incluyendo el límite máximo permitido por la norma americana. El biodiesel en estudio presentó una viscosidad cinemática de $7,932 \pm 0,0260 \mathrm{~mm}^{2} / \mathrm{s}$, que resulta ser alta en comparación con los valores reportados para biodiesel producido de otras materias primas (Demirbas., 2008); se asume que este valor es generado por el gran contenido de esteres metílicos derivados de ácidos grasos saturados y pequeñas contaminaciones de polímeros producidos durante la fritura que no se separaron correctamente en el procedimiento de limpieza, sin embargo los valores de viscosidad y densidad están dentro del límite permitido por la norma ASTM. El valor encontrado para la propiedad número ácido, se encuentra en el límite máximo permitido; esto indica el alto grado de acidez del biodiesel obtenido y consecuentemente podría contribuir a facilitar la corrosión en las partes del motor compuestas por níquel, cobre y latón. El poder corrosivo del biodiesel sobre el metal, es determinado con la ayuda de la corrosión de la tira de cobre, para este caso se obtuvo un valor bajo de 1B, y la norma ASTM permite hasta 3 como límite máximo; esta propiedad es importante para el almacenamiento del combustible. El punto de nube presentado por el biodiesel es de $2,000 \pm 1,000^{\circ} \mathrm{C}$, siendo el principal responsable de este valor el jabón (sales de esteres) producido durante la reacción de transesterificación, porque este solidifica durante el proceso de 
enfriamiento del combustible generando cristales (punto de nube), que pueden contribuir al taponamiento de los filtros y a la pobre inyección del combustible. El valor obtenido para el punto de nube, podría presentar ciertas restricciones para ser utilizado en climas donde se alcancen bajas temperaturas, a diferencia de las regiones cálidas.

Tabla 2: Relación entre las propiedades fisicoquímicas del biodiesel en estudio y la norma ASTM

\begin{tabular}{lccc}
\hline Propiedad fisicoquímica & Método ASTM & $\begin{array}{c}\text { Límite máximo } \\
\text { permitido }\end{array}$ & $\begin{array}{c}\text { Biodiesel de estudio } \\
\mathrm{X} \pm \sigma\end{array}$ \\
\hline Viscosidad Cinemática $20^{\circ} \mathrm{C}$, & ASTM D445 & & \\
$\mathrm{mm}^{2} / \mathrm{s}$ & & $6.500-9.000$ & $7.932 \pm 0,0260$ \\
Densidad $15^{\circ} \mathrm{C}, \mathrm{g} / \mathrm{mL}$ & ASTM D1298 & $0.860-0.900$ & $0.885 \pm 0,010$ \\
Número ácido $\mathrm{mgKOH} / \mathrm{g}$ muestra & ASTM D664 & 0.800 & $0.800 \pm 0,090$ \\
Punto de nube ${ }^{\circ} \mathrm{C}$ & ASTM D2005 & Variable & $2,000 \pm 1,000$ \\
Cantidad de jabón ppm & ASTM D2896 & 500 & $250.000 \pm 12,960$ \\
Corrosión de la tira de cobre & ASTM D130 & 3 & $1 \mathrm{~B}$ \\
\hline
\end{tabular}

Al comparar las figuras 1 y 2 se observan claramente señales caracteristicas del biodiesel que están ausentes en el diesel, como son dos grupos de bandas de absorción propia de los esteres metílicos que lo conforman, así en la región de la huellas dactilares aparece la banda entre (1200$1300) \mathrm{cm}^{-1}$ originada por la deformación axial asimétrica C-O (Pimentel et al., 2006; Lira, 2010) y en la región de los grupos funcionales entre $1750 \mathrm{~cm}^{-1}$ y $1730 \mathrm{~cm}^{-1}$ se encuentra el pico intenso correspondiente al grupo carbonilo $(\mathrm{C}=\mathrm{O})$ propio de los esteres; que está relacionado con la vibración de estiramiento relativamente constante y libre de interferencia, siendo esta señal la mayor diferencia con el espectro del diesel; para ambos espectros es comun la banda de absorción entre (2950 y 3000) $\mathrm{cm}^{-1}$, correspondiente al estiramiento de los enlaces $\mathrm{CH}_{3}, \mathrm{CH}_{2}$ y $\mathrm{CH}$ propio de carbonos alifáticos,

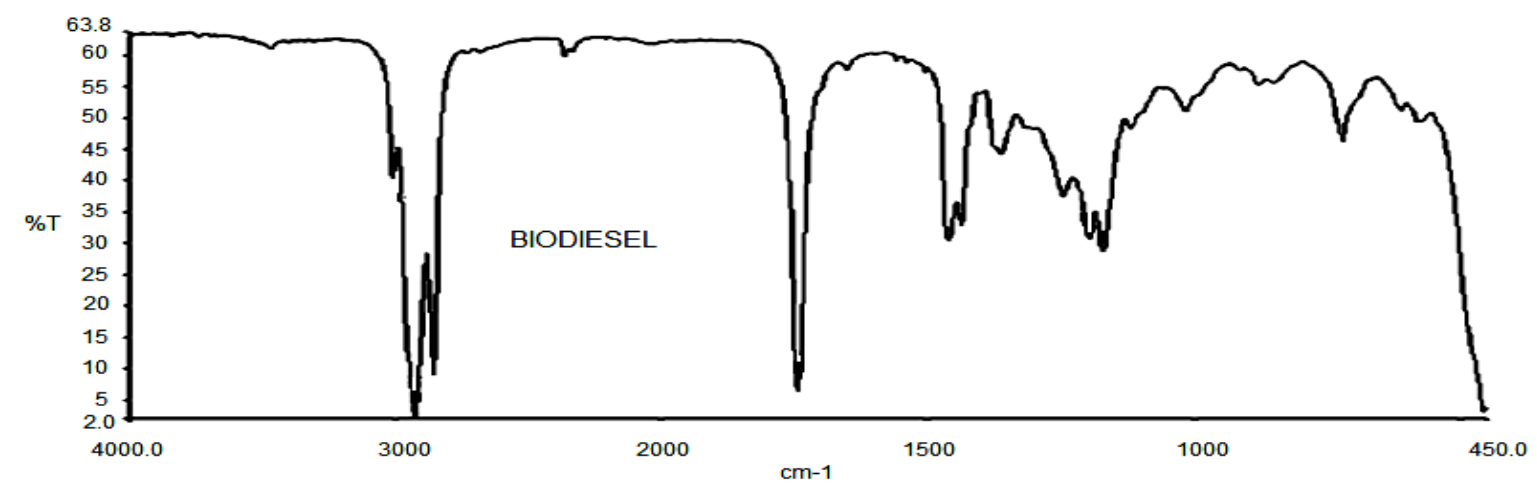

Fig. 1: Espectro infrarrojo del biodiesel de aceite de cocina usado

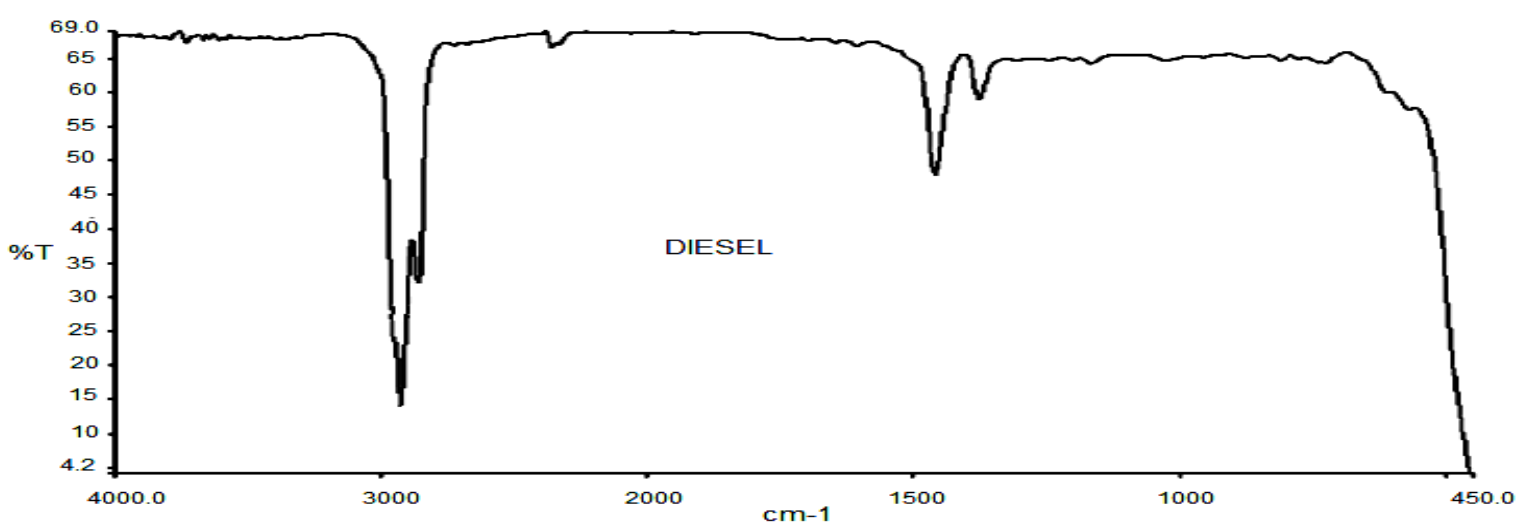

Fig. 2: Espectro infrarrojo del diesel 
Al superponer los espectros de todas las mezclas biodiesel/diesel en el rango de $(0-100) \%$, se encontró una gran diferencia en la altura, absorbancia y area del pico carbonilo; se observó que al aumentar la concentración de biodiesel en las mezclas con diesel la señal del grupo carbonilo presenta un marcado incremento en la absorción. Al realizar el gráfico de la altura del pico carbonilo $\left(\log A_{p}\right)$ en función del logaritmo de la concentración de la muestra analizada ( $\left.\log C\right)$, descrita por la ecuación 1, donde $\mathrm{b}$ es el término independiente y $\mathrm{m}$ la pendiente, en el grafico 3 se observò un comportamiento lineal, con un $\mathrm{R}^{2}=0.9628$ y una desviación estándar poblacional con un valor de 0.03; sin embargo, Aliske utilizando otro software OrinLab-Orin7 encontró para biodiesel de diferentes fuentes valores de $\mathrm{R}^{2}$ cercanos a uno.

$\log A=m \log C+b$

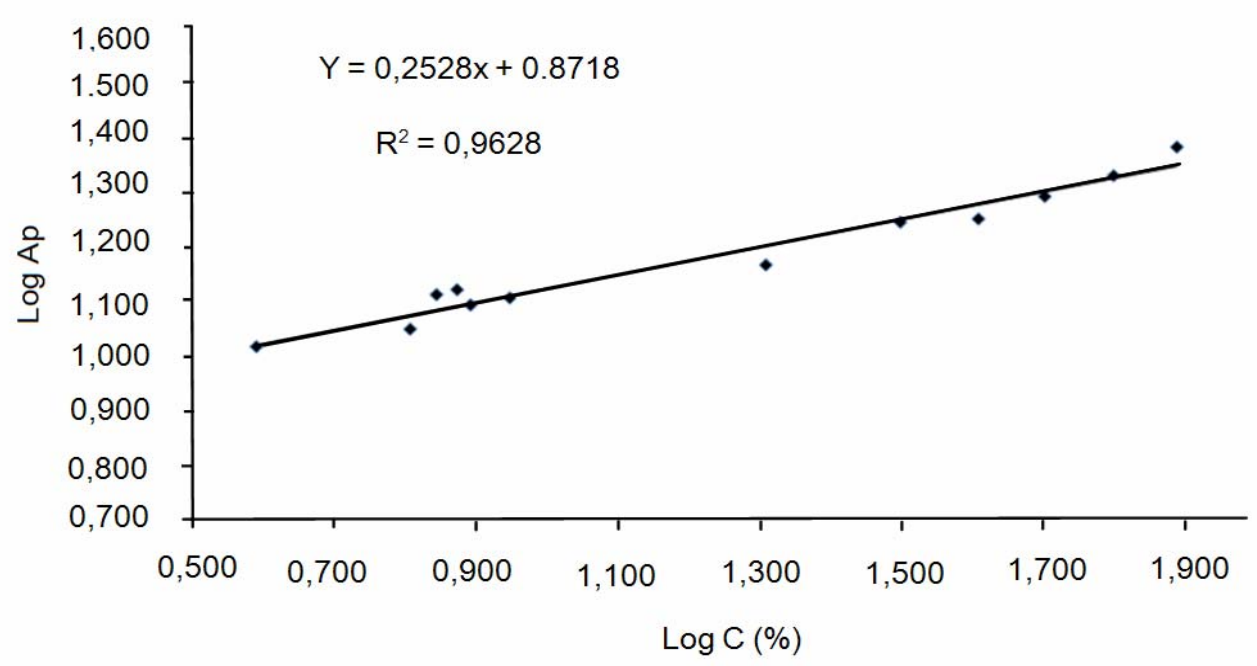

Fig. 3: Variación de la altura del pico con la concentración de las mezclas

Debido a la dispersion en los datos presentada en la figura 3, se ensayó un método alternativo en este trabajo que consistió en graficar la absorbancia del pico carbonilo en función de la concentración de las muestras (ecuación 2). Los resultados obtenidos (figura 4) mostraron un buen ajuste de los datos analíticos obteniéndose una tendencia lineal con $R^{2}$ igual a 0.9987 y una desviación estándar poblacional de 0.001. Es evidente que la segunda metodología produce mejores resultados, observándose incluso una menor dispersión de los datos, por lo que se recomienda emplear este método para cuantificar porcentajes de mezclas biodiesel de aceite de cocina usado/diesel en el intervalo de $5-70 \%$, debido a que valores mas pequeños o mayores de este rango muestran desviación de la linealidad.

$$
A=m C+b
$$

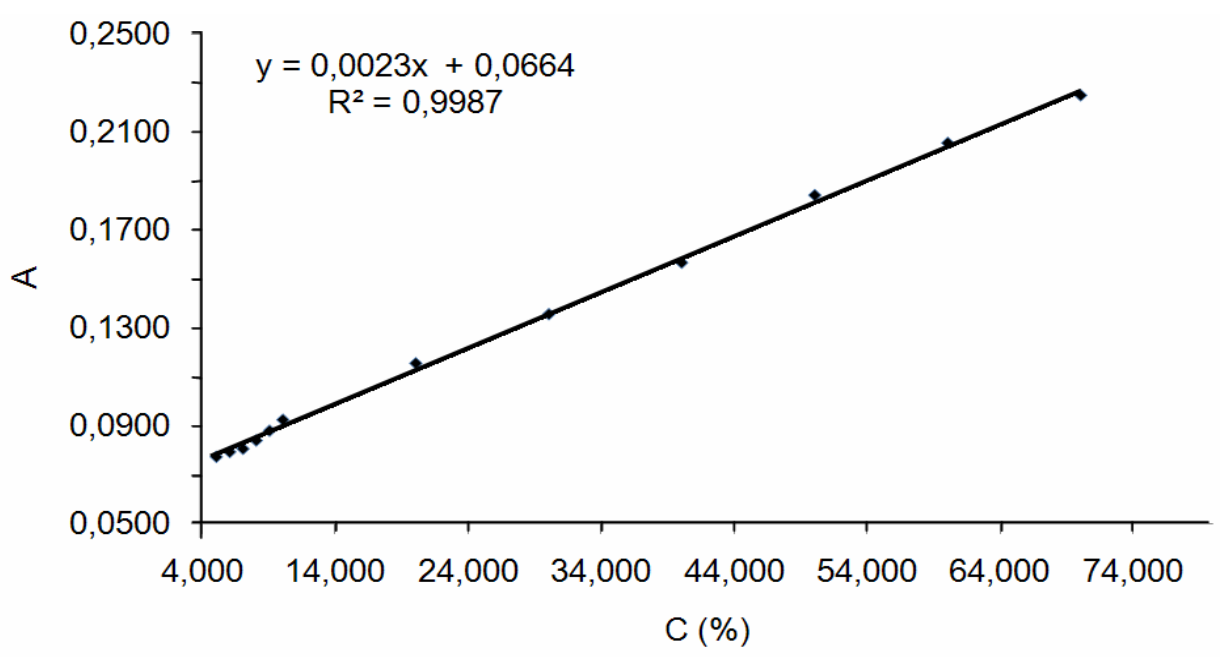

Fig. 4: Variación de la absorbancia del pico carbonilo con la concentración 


\section{CONCLUSIONES}

La proporción molar 1:8 etanol/aceite presentó mayor rendimiento en la preparación del biodiesel a partir de aceite de cocina usado; los espectros de infrarrojo del biodiesel presentaron grandes diferencias con respecto al diesel, mostraron las señales características de los grupos esteres presentes en el biodiesel, como es la señal característica del (C-O) y el pico pronunciado propio del grupo carbonilo $(C=O)$, señales claras libres de interferencia o superposición con el diesel. Con la ayuda del software Spectrum V 2.00, se realizó la interpretación espectral y el análisis cuantitativo de las mezclas biodiesel aceite de cocina usado/diesel en un rango de concentración de $(0-100) \%$ arrojando valores de altura y absorbancia del pico del carbonilo las cuales se incrementaban con el aumento en la concentración de biodiesel en las mezclas con diesel. La espectroscopía infrarroja además de ser una técnica accesible demostró ser una metodología adecuada, para cuantificar los porcentajes de mezcla biodiesel de aceite de cocina usado/diesel en el rango de concentración del (5-70)\%, tomando como patrón el incremento en la absorbancia del grupo carbonilo a medida que aumenta la concentración del biodiesel en las mezclas; se puede decir que en este rango de concentración cumple con la Ley de Beer Lambert.

\section{AGRADECIMIENTOS}

Al Centro de Investigaciones de la Universidad de Córdoba por financiar el proyecto FCB-05-07 numeral 1120235.

\section{REFERENCIAS}

Aliske, M., G. Zagonel., B. Acosta., W. Veiga., C. Saul; Measurement of biodiesel concentration in a diesel oil mixture, Fuel.: 86, 1461-1464 (2007).

Aryee, A., F. Van der Voort., B. Simpson; FTIR determination of free fatty acids in fish oils intended for biodiesel production, Process Biochemistry.: 44, 401-405 (2009).

ASTM D 975-06; Standard specification for diesel fuel oils, (2006).

Dardot , R., R, Augusti., I, Pereira; Electrospray Ionization Mass Spectrometry and Multivariate Calibration Analysis:A Combined Protocol To Quantify Biodiesel in Blends with Petrodiesel, Energy Fuels.: 24, 3183-3188 (2010).

Demirbas, A; Relationship derived from physical properties of vegetable oil and biodiesel fuels, Fuel.: 87, 1743-1748 (2008).

Demirbas A; Biodiesel from waste cooking oil via base-catalytic and supercritical methanol transesterification, Energy Conversion and Management.: 50 923-92 (2009).

EN-590: 2004. Automotive fuels-diesel requeriments and test method, (2004).

Enweremadu, C., M, Mbarawa; Technical aspects of production and analysis of biodiesel from used cooking oil-A review, Renewable and Sustainable Energy Reviews.: 13, 2205-2224 (2009).

Flôres, M., y otros cinco autores; Simultaneous determination of quality arameters of biodiesel/diesel blends using HATR-FTIR spectra and PLS, iPLS or siPLS regressions, Fuel.: 90, 701-706 (2011).

Giraldo, S., Y. Giraldo., L, Ríos., A, Franco., F, Cardeño; Síntesis de Aditivos para Biodiesel a partir de Modificaciones Químicas de la Glicerina, Información Tecnológica.: 20 (6), 75-84 (2009). 
Guarieiro L., A. Pinto ., P, Fernandez., N, Ribeiro; Metodologia analítica para quantificar o teor de biodiesel na mistura biodiesel/diesel utilizando espectroscopia na região do infravermelho, Quim Nova.: 31, 421-6 (2008).

Helwani, Z., M. Othman., N. Aziz., W. Fernando., J. Kim; Technologies for production of biodiesel focusing on green catalytic techniques: A review. Ful Process, Technology.: 90, 1502-1514 (2009).

Kulkarn, M., A. Dalai; Waste cooking oils-an economical source for biodiesel: a review. Industrial \& Engineering Chemistry.: 45, 2901-2913 (2006).

Leuny, D., X. Wu., M. Leung; A review on biodiesel production using catalyzed transesterification, Applied Energy.: 87, 1083-1095 (2010).

Lira, L., y otros cinco autores; Prediction of properties of diesel/biodiesel blends by infrared spectroscopy and multivariate calibration. Fuel.: 89 405-409 (2010).

Marticorena, A., B, Mandagarán., E, Campanella; Análisis del Impacto Ambiental de la recuperación de Metanol en la Producción de Biodiesel usando el Algoritmo de Reducción de Desechos WAR, Información Tecnológica.: 21(1), 23-30 (2010).

Monteiro, M., A. Pepe., L. Morais., A. Ferreira; Critical review on analytical methods for biodiesel characterization, Talanta.: 77, 593-605 (2008).

Oliveiro, J., R. Montalvao., L. Daher., P. Suarez., J. Rubim; Determination of methyl ester contents in biodiesel blends by FTIR-ATR and FTNIR spectroscopies. Talanta.: 69, 1278-1284 (2006).

Pimentel, M., y otros cinco autores; Determination of biodiesel content when blended with mineral diesel fuel using infrared spectroscopy and multivariate calibration, Microchemical Journal.; 82, 201-206 (2006).

Umer, R., A. Farooq; Production of biodiesel through optimized alkaline-catalyzed transesterification of rapeseed oil, Fuel.: 87, 265-273 (2008).

Wang, Y., S. Ou, P. Liu, Z. Zhang; Preparation of biodiesel from waste cooking oil via two-step catalyzed proces, Energy Conversion and Management.: 48, 184-188 (2007). 